\title{
Profile of gantenerumab and its potential in the treatment of Alzheimer's disease
}

This article was published in the following Dove Press journal:

Drug Design, Development and Therapy

12 November 2013

Number of times this article has been viewed

\section{Dijana Novakovic' \\ Marco Feligioni² \\ Sergio Scaccianoce' \\ Alessandra Caruso' \\ Sonia Piccinin ${ }^{2}$ \\ Chiara Schepisi ${ }^{1,2}$ \\ Francesco Errico 3 \\ Nicola B Mercuri ${ }^{4}$ \\ Ferdinando Nicoletti ${ }^{1,5}$ \\ Robert Nisticò ${ }^{1,4}$ \\ 'Department of Physiology and Pharmacology, Sapienza University of Rome, Rome, Italy; ${ }^{2}$ European Brain Research Institute, Rome, Italy; ${ }^{3}$ Ceinge Biotecnologie Avanzate, Naples, Italy; ${ }^{4}$ Laboratory of Experimental Neurology, Istituto di Ricerca e Cura a Carattere Scientifico, Santa Lucia Foundation, Rome, Italy; ${ }^{5}$ Istituto di Ricerca e Cura a Carattere Scientifico, Neuromed, Pozzilli, Italy}

Correspondence: Robert Nisticò Department of Physiology and Pharmacology, Sapienza University of Rome, P Aldo Moro 5, Rome 00I85, Italy Tel +3906501703122

Fax +3906605I3 244

Email robert.nistico@uniromal.it

\begin{abstract}
Alzheimer's disease, which is characterized by gradual cognitive decline associated with deterioration of daily living activities and behavioral disturbances throughout the course of the disease, is estimated to affect 27 million people around the world. It is expected that the illness will affect about 63 million people by 2030, and 114 million by 2050, worldwide. Current Alzheimer's disease medications may ease symptoms for a time but are not capable of slowing down disease progression. Indeed, all currently available therapies, such as cholinesterase inhibitors (donepezil, galantamine, rivastigmine), are primarily considered symptomatic therapies, although recent data also suggest possible disease-modifying effects. Gantenerumab is an investigational fully human anti-amyloid beta monoclonal antibody with a high capacity to bind and remove beta-amyloid plaques in the brain. This compound, currently undergoing Phase II and III clinical trials represents a promising agent with a disease-modifying potential in Alzheimer's disease. Here, we present an overview of gantenerumab ranging from preclinical studies to human clinical trials.
\end{abstract}

Keywords: Alzheimer's disease, gantenerumab, monoclonal antibody, amyloid- $\beta$, clinical trials

\section{Introduction}

Alzheimer's disease (AD) is the most common form of dementia. It is estimated that $\mathrm{AD}$ affects 27 million people around the world, with the number of diagnosed cases expected to rise dramatically in the near future. ${ }^{1} \mathrm{AD}$ is characterized by deficits in memory, language, executive functions, and other intellectual abilities that are serious enough to interfere with daily life. From a neuropathological point of view, the AD brain shows marked atrophy in the brain and the formation of two pathological lesions: extracellular amyloid plaques composed largely of amyloid-beta peptide $(A \beta)$ and neurofibrillary tangles (NFTs), which are intracellular aggregates of hyperphosphorylated tau proteins. ${ }^{2}$ In recent years, however, growing evidence has supported the idea that disruption of connectivity within neural circuits, loss of synapses, and deteriorated synaptic function precedes the death of neurons. At this time, the US Food and Drug Administration and the European Medicines Agency have approved four drugs to treat the cognitive symptoms of AD; three are acetylcholinesterase inhibitors (rivastigmine, galantamine, and donepezil) and the other (memantine) is an uncompetitive antagonist at glutamatergic N-methyl-D-aspartate receptors. Because reduction in the activity of the cholinergic neurons is a well-known feature of AD, acetylcholinesterase inhibitors may improve some cognitive aspects in patients with AD. However, prolonged use of these drugs has also proven effective in slowing down or halting disease progression. 
In fact, several evidences from preclinical studies indicate that these compounds might rescue neuronal damage and death from beta-amyloid (A $\beta$ )-induced toxicity, thus interfering with $\mathrm{AD}$ pathogenesis. ${ }^{3}$ The exact mechanisms by which these effects are achieved remain to be elucidated, although several reports suggest neuroprotective, ${ }^{4,5}$ antiinflammatory, ${ }^{6-8}$ and antioxidant roles. ${ }^{9,10}$ Nevertheless, AD still remains an unmet medical need for which therapies await new discoveries.

\section{Targeting $\mathrm{A} \beta$ production and removal}

At this time, the amyloid cascade hypothesis is the most important theory of $\mathrm{AD}$, postulating that accumulation of $A \beta$ into plaques is the causative pathological event. ${ }^{11}$ On the basis of this hypothesis, interventions that reduce $A \beta$ load in the brain would be likely to attenuate both the neuropathological changes and functional deficits characterizing AD. Indeed, several different $A \beta$-lowering strategies have been developed during past years. Among these, A $\beta$ fibrillogenesis represents a major target for therapeutic intervention in $\mathrm{AD}$ and related human $\beta$-amyloidosis. ${ }^{12}$ Certain small-molecule inhibitors of synthetic $A \beta$ fibrillogenesis inhibit formation of cell-derived, secreted oligomers of $\mathrm{A} \beta$ and prevent the impairment of long-term potentiation (LTP) induced by A $\beta .{ }^{13,14}$ Importantly, this protective effect was achieved only under conditions in which the inhibitors prevented new oligomer formation. ${ }^{15}$ In fact, to be effective, inhibitors of fibrillogenesis need to be used at the initial stages of oligomerization, thus avoiding a paradoxical enhanced neurotoxicity that may derive from active prefibrillar assemblies such as low-n oligomers released after inhibition of fibril formation. For these reasons, a promising strategy consisted of preventing the formation of $A \beta$ by enhancing $\alpha$-secretase activity or inhibiting either $\beta$-secretase or $\gamma$-secretase activity. Among these, either $\gamma$-secretase inhibitors or modulators represented the therapeutic approach with the highest expectations. However, recent clinical trials of $\gamma$-secretase inhibitors and $\gamma$-secretase modulators, including semagacestat, avagacestat, and R-flurbiprofen, have been discontinued for lack of efficacy and/or adverse effects, the mechanisms of which still remain unclear. ${ }^{16}$ An alternative approach consisted of the activation of enzymes or cells that degrade $A \beta$ or $A \beta$ aggregates, thus favoring $A \beta$ clearance. ${ }^{17,18}$ The protease activation strategy is theoretically attractive; however, a lack of specificity and the potential for toxicity confined this approach only to animal modeling studies.

\section{Active versus passive immunotherapy}

The therapeutic potential of clearing $A \beta$ deposition by triggering a humoral immune response to fibrillar $A \beta_{42}$ or passively administering anti-A $\beta$ antibodies has been the most extensively validated anti-A $\beta$ approach in preclinical studies. Either active or passive $A \beta$ immunotherapy was developed to diminish the load of $A \beta$ by promoting its removal. ${ }^{19}$ Active immunization (vaccination) with either $A \beta_{42}$ (the prevalent form of $\mathrm{A} \beta$ in the amyloid plaques of $\mathrm{AD}$ ) or other synthetic fragments has been successfully evaluated in transgenic mouse models of AD and is generally based on the stimulation of T-cell, B-cell, and microglial immune responses. The results of the trials, initially promising, have been partially dashed by the appearance of meningoencephalitis in some patients. ${ }^{20}$ Another type of immunotherapy under investigation implicates passive administration with monoclonal or polyclonal antibodies directed against $A \beta$. This approach circumvents the need for the patient to mount an immunological response to the $\mathrm{A} \beta$ peptide. Although both active and passive administration of anti-A $\beta$ antibodies seem to be effective in reducing $\mathrm{AD}$-like pathology in preclinical models of $\mathrm{AD}$, the mechanism by which they accomplish this effect, whether by stimulating microglial activity, preventing its aggregation or promoting its disaggregation, or a combination of these, still remains elusive.

Of all $A \beta$ immunotherapies, the furthest advanced in clinical development are the passive monoclonal antibodies bapineuzumab ${ }^{21}$ and solanezumab. ${ }^{22}$ Bapineuzumab is a humanized monoclonal antibody against the N-terminus of $\mathrm{A} \beta\left(\mathrm{A} \beta_{1-5}\right)$, whereas solanezumab is a humanized monoclonal antibody designed to bind the central portion of $A \beta\left(A \beta_{12-28}\right)$. Of note, bapineuzumab, despite lowering key biomarkers of $\mathrm{AD}$ such as amyloid brain plaque and phosphorylated tau protein in cerebrospinal fluid, failed to produce significant cognitive improvements in two major clinical trials. ${ }^{23}$ In addition, the Phase III results for solanezumab were only mildly encouraging (Eli Lilly and Co, Indianapolis, IN, USA; Expedition studies 1 and 2), so the development of this compound for the treatment of AD-related dementia will continue with a confirmatory clinical trial to be launched later in 2013 (see the December 12, 2012, press release on the Lilly website). ${ }^{24}$

Other monoclonal antibodies against $\mathrm{A} \beta$ developed so far include PF-04360365 (ponezumab), which targets the free C-terminus of $\mathrm{A} \beta$, and specifically $\mathrm{A} \beta_{34-41}$; MABT5102A, which binds to $A \beta$ monomers, oligomers, and fibrils with equally high affinity; GSK933776A, which, similar to bapineuzumab, targets the $\mathrm{N}$-terminal sequence of $\mathrm{A} \beta$; 
BAN2401, which targets A $\beta$ protofibrils; and gantenerumab, which targets the $\mathrm{N}$-terminus and central portion of $\mathrm{A} \beta$.

\section{Gantenerumab: preclinical studies}

Gantenerumab is a monoclonal immunoglobulin (Ig)G1 antibody selected from a synthetic human combinatorial antibody library (HuCAL ${ }^{\circledR}$; MorphoSys, Martinsried/Planegg, Germany) based on phage display technology and was optimized by in vitro affinity maturation. Different from bapineuzumab and solanezumab, which are humanized versions of murine antibodies, gantenerumab is the first entirely human anti-A $\beta$ monoclonal antibody to enter clinical development.

\section{Pharmacodynamics}

Most of the preclinical data available so far are included in a recently published study. ${ }^{25}$ Gantenerumab passes the blood-brain barrier and has a high binding affinity to cerebral amyloid plaques. The proposed binding mode of gantenerumab to fibrillar $\mathrm{A} \beta$ involves both $\mathrm{N}$-terminal and spatially adjacent central $A \beta$ sequences. According to this model, the flexible $\mathrm{N}$-terminals of $A \beta$ are the initial contact points of gantenerumab binding, followed by interaction with adjacent central $\mathrm{A} \beta$, part of which confers increased binding stability. The specificity of gantenerumab predicts strong binding to native $A \beta$ plaques. Indeed, in brain sections from AD patients and PS2APP transgenic mice, highly efficient immunostaining of fibrillar $A \beta$ was observed at low picomolar concentrations. Equilibrium binding studies, as measured by surface plasmon resonance, indicated dissociation constant $\left(\mathrm{K}_{\mathrm{D}}\right)$ values of $0.6,1.2$, and $17 \mathrm{nM}$ for $A \beta$ fibrils, $A \beta$ oligomers, and $A \beta$ monomers, respectively. Accordingly, long-term treatment with gantenerumab in vivo was associated with a reduction of the amyloid plaque load in PS2APP mice. Notably, no indication for antidrug antibodies was observed during the 5 months of treatment.

One mechanism by which anti-A $\beta$ antibodies might exert their effects is via brain-resident microglial activation. Accordingly, systemic administration of an anti-A $\beta$ antibody binding aggregated $A \beta$ was associated with a marked increase in the number of microglial cells in transgenic AD mice, thus providing insights into the putative mechanism of action of passive immunotherapy for AD. ${ }^{26}$ Similarly, microglia cells and microglia ramifications were spotted in proximity of plaques that reacted with gantenerumab, suggesting a microglia-mediated phagocytic plaque clearance mechanism. Moreover, when cultured with primary human macrophages in vitro, gantenerumab induced cellular phagocytosis of $A \beta$ in human brain tissue. Nevertheless, a possible direct disaggregation of plaques without glial involvement cannot be ruled out. Unlike other $A \beta$ antibodies, gantenerumab interacted only with aggregated $A \beta$ in the brain, without interfering with plasma $A \beta$, indicating that the normal degradation pathway of circulating $A \beta$ is not affected. Moreover, gantenerumab neither triggered reactive inflammation on degradation of brain plaques nor worsened the occurrence of cerebral $A \beta$ angiopathy or microhemorrhages.

\section{Pharmacokinetics}

Similar linear pharmacokinetics profiles of gantenerumab were obtained in both wild-type and transgenic models of AD. ${ }^{25}$ Accordingly, in both wild-type and PS2APP mice, gantenerumab exhibited a biphasic appearance with approximately dose-proportional increases in maximum concentration $\left(\mathrm{C}_{\max }\right)$ and area-under-the-curve parameters. Moreover, gantenerumab was eliminated slowly in transgenic or wild-type mice. To establish the kinetic profile of gantenerumab binding in vivo in the PS2APP model of $A D$, its reactivity with $A \beta$ plaques was measured for up to 9 weeks. The maximal binding of gantenerumab to $A \beta$ plaques was generally observed at different doses after 2-3 weeks in all brain regions with plaques (ie, cortex, hippocampus, and thalamus). The binding of gantenerumab to $A \beta$ plaques was still measurable for more than 2 months, as shown for plaques in the frontal cortex, whereas the half-life of gantenerumab in plasma was 6.5 days and was no longer detectable after 3 weeks. ${ }^{25}$

\section{Functional studies}

During the last few years, transgenic models have offered the advantage of examining the bases for the spatial/temporal evolution of the disease. In addition, the combination of electrophysiological and behavioral techniques has been a very powerful tool not only to address important questions about the pathogenic mechanisms of the disease but also to test the functional outcome of novel ligands ${ }^{27,28}$ and assess the efficacy of therapies for the treatment and/or prevention of $\mathrm{AD}$ and other neurodegenerative conditions. ${ }^{29}$ LTP, which represents a widely accepted model of learning and memory at the cellular level, is typically altered after manipulation of specific plasticity-related genes ${ }^{30-33}$ and is impaired in several animal models of human neuropsychiatric disorders, such as Parkinson's disease, ${ }^{34}$ depression, ${ }^{35}$ autism spectrum disorders, ${ }^{36}$ and multiple sclerosis. ${ }^{37}$ Most currently studied AD models show cognitive deficits and age-related disruption of synaptic markers and amyloid plaque deposition, but few strains show evidence of significant cell death. Most studies have reported, principally, either inhibition of LTP 
or reduction in baseline fast excitatory transmission before plaque deposition. ${ }^{38,39}$

Interventions that reduce $A \beta$ load in the brain would be likely to attenuate both the neuropathological changes and functional deficits characterizing AD. Indeed, lowering the production of the peptide by inhibiting the enzymes responsible for $A \beta$ generation, preventing the formation of $A \beta$ aggregates, and increasing the rate of $A \beta$ clearance from the brain have all proven successful in experimental models of AD. ${ }^{11}$ Accordingly, preclinical studies using 3D6 (the murine form of bapineuzumab) have demonstrated resolution of $A \beta$ plaque and preservation of LTP and behavioral deficit in different transgenic A $\beta$ PP models..$^{40,41}$ Notably, 3D6 was shown to bind to soluble, synaptotoxic assemblies of $A \beta$, thus preventing $A \beta$ downstream effects. In addition, acute and subchronic treatment with solanezumab was able to attenuate memory deficits in platelet-derived growth factor promoter expressing amyloid precursor protein transgenic mice, ${ }^{42}$ having lesser hemorrhagic potential than bapineuzumab. Along these lines, the ability of gantenerumab to react not only with the $A \beta$ fibrils and plaques but also with oligomeric $A \beta$ might suggest its potential in restoring synaptic deficits associated with AD models. Indeed, in vivo measurements in adult male Sprague Dawley rats showed that gantenerumab attenuated the inhibition of LTP caused by intracerebroventricular injection of soluble $\mathrm{A} \beta_{42}$ aggregates. The effects of gantenerumab on cognitive assessment in the Morris water maze test were inconclusive, as both wild-type and transgenic $\mathrm{AD}$ mice injected with vehicle displayed impairment of learning, which might be ascribed to stress conditions caused by weekly intracerebroventricular injections. Importantly, neurological or motor impairments were not detected even after 5 months of treatment. ${ }^{24}$

\section{Clinical studies}

\section{Phase I}

\section{NCT0053 I 804}

Safety, tolerability, pharmacokinetic, and pharmacodynamic profiles of gantenerumab were investigated in a multicenter, multiple-ascending dose, randomized, double-blind, placebocontrolled and parallel-group study. Gantenerumab was administered through intravenous infusion in patients with mild to moderate $\mathrm{AD}$.

The study is now completed ${ }^{43}$ and included data from $16 \mathrm{AD}$ patients, aged $50-90$ years, who were also included in a positron emission tomographic (PET) substudy of a larger multiple ascending dose trial. For inclusion in the study the patients had probable AD according to the National Institute of
Neurological Disorders and Stroke-Alzheimer's Disease and Related Disorders Association criteria, a Mini-Mental State Examination Score between 16 and 26 (inclusive), a magnetic resonance imaging scan consistent with $\mathrm{AD}$, and a modified Hachinski ischemia score of 4 or less. Apolipoprotein E (APOE) genotyping was also performed for all patients.

The patients were assigned to receive intravenous infusions of gantenerumab ( $60 \mathrm{mg}, \mathrm{n}=6 ; 200 \mathrm{mg}, \mathrm{n}=6$ ) or placebo $(n=4)$ once every 4 weeks to a total of seven infusions, but because of early termination of dosing in the 200-mg gantenerumab group, not all participants received seven infusions. Baseline images were compared with images obtained at the end of treatment to determine the change in brain amyloid, as measured by carbon $11\left[{ }^{11} \mathrm{C}\right]$-labeled Pittsburgh compound B PET. In addition, to evaluate gantenerumab's ability to clear amyloid plaques via phagocytosis, primary microglial cells obtained from healthy human brain tissue during tumor surgery were incubated in different concentrations of the drug. Sixteen patients with end-of-treatment PET scans were included in the analysis. The observed mean treatment difference versus placebo ( $95 \%$ confidence interval) in cortical brain amyloid level was $-15.6 \%$ for the 60 -mg group and $-35.7 \%$ for the $200-m g$ group. Notably, changes were generally steady across brain regions with amyloid deposits. No consistent treatment effects on cognitive endpoints were revealed in this small group of patients treated for a short period of time. In any case, cognitive measures did not correlate with a reduction in amyloid burden. Findings in the placebo group supported previous reports that amyloid load progressively increases in many patients with mild to moderate $\mathrm{AD}$. Two patients treated with gantenerumab, both of them APOE $\varepsilon 4$ homozygous, showed abnormalities on magnetic resonance imaging fluid-attenuated inversion recovery imaging that were "consistent with inflammation or vasogenic edema" after two and four infusions of the 200-mg dose group. Both patients also developed microhemorrhages, and one was "mildly symptomatic," with headaches, dizziness, gait instability, and tremor. These adverse effects resolved spontaneously after discontinuation of dosing, similar to what has been reported after treatment with bapineuzumab. The fluid-attenuated inversion recovery abnormalities were most conspicuous in areas of more prominent amyloid reduction and may provide clues as to the mechanism by which gantenerumab reduces amyloid. It is known that microglial cells contain very low levels of $A \beta$ in untreated patients with $A D$, whereas postmortem studies after treatment with AN-1792 suggest that antiamyloid antibodies lead to an increase in A $\beta$ phagocytosis. ${ }^{44,45}$ Indeed, live-cell imaging indicated 
that a clearance of fluorescent-labeled gantenerumab bound to amyloid deposits occurred in a dose-dependent manner within hours via active intracellular uptake by brain-activated microglia adjacent to amyloid plaques.

\section{Phase II NCT0I760005}

The aim of this study is to assess the safety, tolerability, and biomarker efficacy of gantenerumab versus solanezumab in individuals who have an autosomal dominant Alzheimer's disease mutation. This is a randomized, double-blind, placebo-controlled, multicenter study that will analyze two potential diseases and the related therapeutic protocols in individuals at risk for and with dominantly inherited AD (individuals who are known to have disease, resulting from a mutation, or were unaware of their genetic status). This study will enroll 210 participants who will receive gantenerumab at the dose of $225 \mathrm{mg}$ subcutaneously every 4 weeks or solanezumab at the dose of $400 \mathrm{mg}$ with intravenous infusions every 4 weeks. This study design will include a placebo shared by all treatment groups. The primary outcome of the study is the amount of fibrillar amyloid deposition as measured by $\left[{ }^{11} \mathrm{C}\right]$ PiB-PET scans at baseline and after 2 years of treatment. The estimated study completion date is December 2016.

\section{Phase III \\ NCTOI224106}

This is a multicenter, randomized, double blind, placebocontrolled, and parallel group study that will evaluate the effect of gantenerumab on cognition and mental functioning in patients with AD. This study will assess whether gantenerumab can help prevent the symptoms of prodromal AD from getting worse. This study should include 770 patients aged 50-85 years who will receive gantenerumab subcutaneously at the dose of $225 \mathrm{mg}$ or $105 \mathrm{mg}$ every 4 weeks for 104 weeks. The estimated study completion date is September 2016.

\section{Conclusions}

According to the amyloid- $\beta$ hypothesis of AD, several monoclonal anti-A $\beta$ antibodies with significant amyloid-lowering effects have been successfully tested in preclinical models of AD. Some of them, such as bapineuzumab and solanezumab, have been humanized, whereas gantenerumab represents the first fully human antibody in clinical development as a potential disease-modifying therapy. Human, rather than xenogenic, antibodies are the preferred choice for long-term administration because they are associated with a low risk of production of neutralizing antibodies. Moreover, although all of the $A \beta$ antibodies described so far, including those in clinical testing, typically bind to one region of the $A \beta$ sequence, gantenerumab binds to both $\mathrm{N}$-terminal and central regions of $A \beta$ in a configuration not present in the structure of monomeric $A \beta$, which is believed to exert neuroprotective activity. ${ }^{46}$ Thus, gantenerumab represents a potent anti-A $\beta$ antibody with a preference for aggregated $A \beta$.

At present, it remains unclear whether any reduction in brain amyloid level will successfully translate into clinical efficacy. It has to be taken into account that setbacks with passive and active immunotherapies are not uncommon, and actually they represent the rule rather than the exception. Importantly, the scientific basis of these treatments is solid, and number of novel therapeutic approaches will soon enter the clinical pipeline.

\section{Acknowledgments}

We thank Dr Pignatelli for critical reading of the manuscript. $\mathrm{RN}$ is supported by grants from the Ministry of Education, University and Research.

\section{Disclosure}

The authors report no conflicts of interest in this work.

\section{References}

1. Thies W, Bleiler L; Alzheimer's Association. 2013 Alzheimer's disease facts and figures. Alzheimers Dement. 2013;9(2):208-245.

2. Selkoe DJ. Alzheimer's disease is a synaptic failure. Science. 2002;298(5594):789-791.

3. Francis PT, Nordberg A, Arnold SE. A preclinical view of cholinesterase inhibitors in neuroprotection: do they provide more than symptomatic benefits in Alzheimer's disease? Trends Pharmacol Sci. 2005;26(2): 104-111.

4. Zhou J, Fu Y, Tang XC. Huperzine A and donepezil protect rat pheochromocytoma cells against oxygen-glucose deprivation. Neurosci Lett. 2001;306(1-2):53-56.

5. Wang ZF, Zhou J, Tang XC. Huperzine B protects rat pheochromocytoma cells against oxygen-glucose deprivation-induced injury. Acta Pharmacol Sin. 2002;23(12):1193-1198.

6. Tabet N. Acetylcholinesterase inhibitors for Alzheimer's disease: anti-inflammatories in acetylcholine clothing! Age Ageing. 2006;35(4): 336-338.

7. Shytle RD, Mori T, Townsend K, et al. Cholinergic modulation of microglial activation by alpha 7 nicotinic receptors. $J$ Neurochem. 2004;89(2):337-343.

8. Pollak Y, Gilboa A, Ben-Menachem O, Ben-Hur T, Soreq H, Yirmiya R Acetylcholinesterase inhibitors reduce brain and blood interleukin-1beta production. Ann Neurol. 2005;57(5):741-745.

9. Xiao XQ, Wang R, Tang XC. Huperzine A and tacrine attenuate beta-amyloid peptide-induced oxidative injury. J Neurosci Res. 2000;61(5):564-569.

10. Klugman A, Naughton DP, Isaac M, Shah I, Petroczi A, Tabet N. Antioxidant enzymatic activities in Alzheimer's disease: the relationship to acetylcholinesterase inhibitors. J Alzheimers Dis. 2012;30(3): $467-474$. 
11. Hardy J, Selkoe DJ. The amyloid hypothesis of Alzheimer's disease: progress and problems on the road to therapeutics. Science. 2002; 297(5580):353-356.

12. Findeis MA. Peptide inhibitors of beta amyloid aggregation. Curr Top Med Chem. 2002;2(4):417-423.

13. Nakagami $Y$, Nishimura $\mathrm{S}$, Murasugi T, et al. A novel compound RS-0466 reverses beta-amyloid-induced cytotoxicity through the Akt signaling pathway in vitro. Eur J Pharmacol. 2002;457(1):11-17.

14. Nakagami Y, Nishimura S, Murasugi T, et al. A novel beta-sheet breaker, RS-0406, reverses amyloid beta-induced cytotoxicity and impairment of long-term potentiation in vitro. $\mathrm{Br} J$ Pharmacol. 2002;137(5):676-682.

15. Walsh DM, Klyubin I, Shankar GM, et al. The role of cell-derived oligomers of Abeta in Alzheimer's disease and avenues for therapeutic intervention. Biochem Soc Trans. 2005;33(Pt 5):1087-1090.

16. Toyn JH, Rowley A, Matsuoka Y, Tomita T, Imbimbo BP. $\gamma$-Secretase Pharmacology: What Pharmacology Will Work for Alzheimer's Disease? Int J Alzheimers Dis. 2013;2013:849128.

17. Leissring MA. Proteolytic degradation of the amyloid beta-protein: the forgotten side of Alzheimer's disease. Curr Alzheimer Res. 2006;3(5): $431-435$.

18. Selkoe DJ. Clearing the brain's amyloid cobwebs. Neuron. 2001; 32(2):177-180.

19. Schenk D. Amyloid-beta immunotherapy for Alzheimer's disease: the end of the beginning. Nat Rev Neurosci. 2002;3(10):824-828.

20. Senior K. Dosing in phase II trial of Alzheimer's vaccine suspended. Lancet Neurol. 2002;1(1):3.

21. Kerchner GA, Boxer AL. Bapineuzumab. Expert Opin Biol Ther. 2010;10(7):1121-1130.

22. Samadi H, Sultzer D. Solanezumab for Alzheimer's disease. Expert Opin Biol Ther. 2011;11(6):787-798.

23. Vellas B, Carrillo MC, Sampaio C, et al; EU/US/CTAD Task Force Members. Designing drug trials for Alzheimer's disease: what we have learned from the release of the phase III antibody trials: a report from the EU/US/CTAD Task Force. Alzheimers Dement. 2013;9(4):438-444.

24. Eli Lilly and Company. Lilly provides update on next stepsfor Solanezumab; December 12, 2012. Available from: http://newsroom. lilly.com/releasedetail.cfm?ReleaseID=726309. Accessed October 20, 2013.

25. Bohrmann B, Baumann K, Benz J, et al. Gantenerumab: a novel human anti-A $\beta$ antibody demonstrates sustained cerebral amyloid- $\beta$ binding and elicits cell-mediated removal of human amyloid- $\beta$. J Alzheimers Dis. 2012;28(1):49-69.

26. Koenigsknecht-Talboo J, Meyer-Luehmann M, Parsadanian M, et al. Rapid microglial response around amyloid pathology after systemic anti-Abeta antibody administration in PDAPP mice. $J$ Neurosci. 2008;28(52):14156-14164.

27. Dolman NP, Troop HM, More JC, et al. Synthesis and pharmacology of willardiine derivatives acting as antagonists of kainate receptors. J Med Chem. 2005;48(24):7867-7881.

28. Dargan SL, Clarke VR, Alushin GM, et al. ACET is a highly potent and specific kainate receptor antagonist: characterisation and effects on hippocampal mossy fibre function. Neuropharmacology. 2009;56(1): 121-130.

29. Nisticò R, Pignatelli M, Piccinin S, Mercuri NB, Collingridge G. Targeting synaptic dysfunction in Alzheimer's disease therapy. Mol Neurobiol. 2012;46(3):572-587.

Drug Design, Development and Therapy

\section{Publish your work in this journal}

Drug Design, Development and Therapy is an international, peerreviewed open-access journal that spans the spectrum of drug design and development through to clinical applications. Clinical outcomes, patient safety, and programs for the development and effective, safe, and sustained use of medicines are a feature of the journal, which
30. Errico F, Nisticò R, Palma G, et al. Increased levels of d-aspartate in the hippocampus enhance LTP but do not facilitate cognitive flexibility. Mol Cell Neurosci. 2008;37(2):236-246.

31. Errico F, Nisticò R, Napolitano F, et al. Increased D-aspartate brain content rescues hippocampal age-related synaptic plasticity deterioration of mice. Neurobiol Aging. 2011;32(12):2229-2243.

32. Errico F, Nisticò R, Napolitano F, et al. Persistent increase of D-aspartate in D-aspartate oxidase mutant mice induces a precocious hippocampal age-dependent synaptic plasticity and spatial memory decay. Neurobiol Aging. 2011;32(11):2061-2074.

33. Molinaro P, Viggiano D, Nisticò R, et al. Na+-Ca2+ exchanger (NCX3) knock-out mice display an impairment in hippocampal long-term potentiation and spatial learning and memory. J Neurosci. 2011;31(20): 7312-7321.

34. Bonito-Oliva A, Pignatelli M, Spigolon G, et al. Cognitive Impairment and Dentate Gyrus Synaptic Dysfunction in Experimental Parkinsonism. Biol Psychiatry. Epub March 28, 2013.

35. Pignatelli M, Vollmayr B, Richter SH, et al. Enhanced mGlu5-receptor dependent long-term depression at the Schaffer collateral-CA1 synapse of congenitally learned helpless rats. Neuropharmacology. 2013;66: 339-347.

36. Pignatelli M, Feligioni M, Piccinin S, Molinaro G, Nicoletti F, Nisticò R. Synaptic plasticity as a therapeutic target in the treatment of autism-related single-gene disorders. Curr Pharm Des. Epub February 13, 2013.

37. Nisticò R, Mango D, Mandolesi G, et al. Inflammation subverts hippocampal synaptic plasticity in experimental multiple sclerosis. PLoS One. 2013;8(1):e54666.

38. Balducci C, Mehdawy B, Mare L, et al. The $\gamma$-secretase modulator CHF5074 restores memory and hippocampal synaptic plasticity in plaque-free Tg2576 mice. J Alzheimers Dis. 2011;24(4):799-816.

39. La Rosa LR, Matrone C, Ferraina C, et al. Age-related changes of hippocampal synaptic plasticity in A $\beta \mathrm{PP}$-null mice are restored by NGF through p75 NTR. J Alzheimers Dis. 2013;33(1):265-272.

40. Knobloch M, Farinelli M, Konietzko U, Nitsch RM, Mansuy IM. Abeta oligomer-mediated long-term potentiation impairment involves protein phosphatase 1-dependent mechanisms. J Neurosci. 2007; 27(29):7648-7653.

41. Zago W, Buttini M, Comery TA, et al. Neutralization of soluble, synaptotoxic amyloid $\beta$ species by antibodies is epitope specific. J Neurosci. 2012;32(8):2696-2702.

42. Dodart JC, Bales KR, Gannon KS, et al. Immunization reverses memory deficits without reducing brain Abeta burden in Alzheimer's disease model. Nat Neurosci. 2002;5(5):452-457.

43. Ostrowitzki S, Deptula D, Thurfjell L, et al. Mechanism of amyloid removal in patients with Alzheimer disease treated with gantenerumab. Arch Neurol. 2012;69(2):198-207.

44. Boche D, Nicoll JA. The role of the immune system in clearance of Abeta from the brain. Brain Pathol. 2008;18(2):267-278.

45. Nicoll JA, Barton E, Boche D, et al. Abeta species removal after abeta42 immunization. $J$ Neuropathol Exp Neurol. 2006;65(11): $1040-1048$

46. Giuffrida ML, Caraci F, Pignataro B, et al. Beta-amyloid monomers are neuroprotective. J Neurosci. 2009;29(34):10582-10587.

has also been accepted for indexing on PubMed Central. The manuscript management system is completely online and includes a very quick and fair peer-review system, which is all easy to use. Visit http://www.dovepress.com/testimonials.php to read real quotes from published authors. 